\title{
FIELD EVALUATION OF TALSTAR (BIFENTHRIN) RESIDENTIAL BARRIER TREATMENTS ALONE AND IN CONJUNCTION WITH MOSQUITO MAGNET LIBERTY PLUS TRAPS IN CEDAR KEY, FLORIDA
}

\author{
AARON M. LLOYD', DANIEL L. KLINE², AND ULRICH BERNIER ${ }^{2}$ \\ 'Lee County Mosquito Control District, 15191 Homestead Rd. \\ Lehigh Acres, FL. 33905 \\ Lloyd@lcmcd.org:
}

${ }^{2}$ USDA, ARS, CMAVE, 1600-1700 SW 23 ${ }^{\text {rd }}$ Dr. Gainesville, FL. 32608

Dan.kline@usda.gov

Subiect Editor: Rui-De Xue

\begin{abstract}
The effectiveness of bifenthrin applications to vegetation with and without commercial mosquito traps (Mosquito Magnet Liberty Plus) was evaluated against Culicoides biting midges in a residential coastal area located in Cedar Key, Florida. Efficacy evaluations were determined by surveillance trap collections and modified landing rate counts. In general, all treatments provided significant reduction from Culicoides biting midge pressure when compared with untreated yards with no traps (control). However, the combination of bifenthrin and Liberty Plus traps proved to be the most successful in reducing Culicoides compared with yards with only a Liberty Plus trap. Yards treated with bifenthrin alone or in combination with the Liberty Plus trap were more successful than controls, suggesting that Culicoides biting midge population suppression may be obtained through barrier application alone.

Key Words: Culicoides, biting midges, bifenthrin, barrier, traps
\end{abstract}

\section{INTRODUCTION}

Insecticides when applied as barrier sprays to vegetation has proven effective in the suppression of mosquitoes (Fulcher et al. 2015) and can be an effective part of an Integrated Mosquito Management plan (Richards et al. 2017). However, such applications have limited success against Culicoides biting midges. Kettle (1949) attempted to control Culicoides impunctatus (Goetghebuer) in Scotland by providing a barrier spray of DDT to vegetation at two pounds per acre. Unfortunately, this effort provided no control. Previous studies have reported success with fogging applications of organophosphates or DDT (Trapido 1947, Bruce and Blakeslee 1948). Conversely, Linley and Davies (1971) concluded that fogging was a waste of effort best reserved for emergencies when a Culicoides population reaches intolerable levels.

Biting midge suppression has been shown when adulticiding aerially using ultra low volume (ULV) applications (Breidenbaugh and Szalay 2010). However, these applications are limited to equipment availability, operating costs, and duration of effectiveness. Madden et al. (1946) were able to control C. furens (Poey) for three days using $0.28 \mathrm{~kg}$ DDT per hectare when applied aerially. Giglioli et al. (1980) reported 95\% reduction when applying ULV fenitrothion aerially. Haile et al. (1984) reported that aerial ULV applications with naled provided $99 \%$ control for up to three days; the authors concluded that Culicoides control could be obtained with sufficient dose and frequency using this product. Linley and Jordan (1992) were able to provide $90 \%$ control for adult 
Culicoides populations when applying insecticide using an aerial ULV machine.

The pesticide industry has moved to more environmentally safe pesticides such as pyrethroids to accommodate Environmental Protection Agency (EPA) regulations. Furthermore, tests have shown that Culicoides have a higher mortality rate when exposed to pyrethroids than organophosphates (Kline et al. 1981, Floore 1985). This suggests that the use of pyrethroids could be a vital part of today's IPM program for Culicoides. Standfast et al. (2003) used bifenthrin to treat various home external resting surfaces in River Heads, Hervey Bay, Queensland, Australia. Applied as a coarse spray, bifenthrin resulted in a 75 to $97 \%$ reduction in biting midge numbers in the first month and a $65 \%$ reduction at 6 weeks post-treatment. The authors concluded that the bifenthrin applications were successful in controlling biting midge numbers during peak emergence while satisfying federal concerns about broad-scale insecticide application to sensitive areas.

Adult mosquito traps are commonly used for mosquito surveillance to evaluate treatment methods or monitor mosquito population abundance (WHO 2013, Li et al. 2016, Bazin and Williams 2018, Wilke et al. 2019)). Mosquito traps have also been used with some success as a control technique to reduce mosquito populations using a propane fueled Mosquito Magnet (MM) Pro (Kline 2006). Moreover, mosquito trap collections have recorded large numbers of Culicoides biting midges in the capture nets of MM Freedom and Liberty Plus traps (Lloyd et al. 2008) posing the question; can mosquito traps, alone, be used to reduce Culicoides biting midge populations?

The objective of this study was to evaluate the efficacy of treating shrubbery surrounding homes with Talstar (7.9\% bifenthrin) only or MM Liberty Plus traps alone as well as their combination against Culicoides populations

Study Site. The study site consisted of a residential neighborhood (Rye Key; 29.153982, -83.0460009) in Cedar Key, Florida. Rye Key is a 5.91 ha is- land surrounded by the Gulf of Mexico with extensive inlets with black needle rush (Juncus spp.) and cordgrass (Spartina spp.).marsh located at the northeastern tip of Cedar Key. This site was chosen because of its previous history of consistently producing large populations of Culicoides (Lloyd et al. 2008). In addition, access to the neighborhood was limited by an electronic gate reducing the chance of vandalism or theft of equipment. The surrounding flora associated with each site was similar.

Treatments. Treatment one was a MMLiberty Plus ${ }^{\circledR}$ trap (Liberty Plus), baited with an octenol cartridge manufactured by Wood Stream Corporation (Lititz, PA). The Liberty Plus trap was set up per manufacturers recommendations to run continuously during the study. The Liberty Plus is a propane powered, counterflow geometry trap that encapsulates a hybrid power fueled by propane that generates heat, moisture, and approximately $550 \mathrm{ml} / \mathrm{min}$ combusted $\mathrm{CO}_{2}$. The average surface temperature of this trap was $37.1^{\circ} \mathrm{C}$ with plume temperatures between 33.3 to $40.6{ }^{\circ} \mathrm{C}$. The Liberty Plus has a pushbutton start with lights that indicate when the machine is operating and if service is needed.

Treatment two was a Talstar ${ }^{\circledR}(7.9 \%$ bifenthrin) (FMC, Philadelphia, PA) application to shrubbery surrounding the home. The insecticide was applied at max label rate (29.6 milliliters per 3.8 liters, 3.8 liters per 92.9 square meters) using a Solo® (Newport News, VA) backpack sprayer delivering the insecticide dilution in low volume. Retreatment of applications following manufacturer's label instructions of once every four weeks. Treatment three was a combination of the Liberty Plus trap as described in treatment one and Talstar application as described in treatment two. Treatment four was a control with locations under normal 
conditions without traps or insecticide. The study was conducted between March and October 2009. All residences in the study were at least $50 \mathrm{~m}$ from one another.

Data Collection. Two different assessments were incorporated during the study at each residential location. One surveillance sticky trap was placed at each location between the house and treatment. Traps were constructed of a $15.2 \times 15.2 \mathrm{~cm}$ sheet of Web Trap ${ }^{\circledR}$ adhesive papers (Applica, Miami Lakes, FL) mounted onto a wooden stake secured $1 \mathrm{~m}$ above the ground and baited with $0.45 \mathrm{~kg}$ of dry ice housed in a cooler on the ground (John W. Hock, Gainesville, FL). Collections were obtained every 1-2 days to record biting midge capture for that time period. The surveillance sticky trap was baited with dry ice every 1-2 days and placed at the location before dusk. A flyswatter count was taken every 1-2 days per week during the study at each residential location for 3 minutes using a $15.2 \mathrm{x}$ $15.2 \mathrm{~cm}$ section cut from a sheet of Web Trap ${ }^{\circledR}$ adhesive paper (Applica, Miami Lakes, FL) and used to swat pursuing Culicoides from the air.

The Liberty Plus trap nets were collected and replaced with a replacement net 1-2 days per week throughout the study. Each Liberty Plus net removed from traps were placed into individual one-gallon plastic Ziploc bag, and any insects inside traps but outside nets were vacuumed and placed inside the respective Ziploc bag. Each Ziploc bag was labeled individually with location, date, and trap identifier. The propane tanks were changed every 18 days. The octenol cartridges were changed every 21 days.

Swatting count were conducted every 1-2 days per week from each location, covered in clear cellophane wrap and labeled with location, date, and treatment information. Surveillance sticky papers were collected every 1-2 days per week from each location and replaced with a new paper. The collected surveillance sticky papers were covered in clear cellophane wrap and labeled with location, date, and treatment information.

Once collections were returned to the laboratory they were stored in a $-20{ }^{\circ} \mathrm{C}$ freezer until processed. If the number of Culicoides captured was estimated to be more than 500 , an aliquot was extrapolated from the total capture and weighed. The weight of the aliquot was divided into the total captured weight and the quotient was multiplied by the number of Culicoides identified and counted in the aliquot. If the number of Culicoides was estimated to be below 500, the entire collection was identified and counted. Samples were identified to species and counted (Blanton and Wirth 1979).

Data Analysis. Data were initially normalized by conversion to $\log _{10}(n+1)$ then subjected to ANOVA (SAS 2003) using the following model statements: Method = Swatting Treatment Week; Method = Sticky Treatment Week; Treatment $=$ Control Method Week; Treatment = Liberty Plus Method Week; Treatment = Liberty Plus/ Talstar Method Week; Treatment $=$ Talstar Method Week; where dependant variables represented numbers of biting midges captured. Method was one of the surveillance methods used to determine biting midge pressure, treatment was one of the four assigned control measures, and week was one of the 20 trapping weeks of the study. Means were separated with the Ryan-Einot-GabrielWelsch Multiple Range Test (REGWQ), and unless otherwise stated, $\mathrm{P}<0.05$ (SAS 2003). Although $\log _{10}(n+1)$ values were used for the analyses, actual means are reported in the text, and tables.

\section{RESULTS}

Analysis of data by calendar week yielded no significant difference among treatments and weeks for all Culicoides, C. furens, and C. mississippiensis captured. Significant differences in the sticky surveillance and swatting count methods used to survey all Culicoides from March to October 2009 were observed $(\mathrm{F}=18.10, \mathrm{df}=3,19, \mathrm{p}<0.0004)$. Sticky sur- 
veillance trap method consistently captured more Culicoides than the swatting counts.

Four species of Culicoides: C. barbosai (Wirth and Blanton), C. furens Poey, $C$. melleus (Coquillett), and C. mississippiensis Hoffman were collected from sticky traps, flyswatters, and MM Liberty Plus traps (Tables $4-1,2)$. There was no significant difference among treatments and weeks or surveillance methods and weeks when overall total abundance was considered on sticky traps. However, all treatments, significantly reduced total abundance of midges compared with controls from those traps (Table 4-3). However, total Culicoides reduction was not significantly different between Talstar application with and without the Liberty Plus. Two major species (C. furens and C. mississippiensis) were collected in large enough numbers to be statistically analyzed. Culicoides furens reduction was similar to that for previously mentioned for total Culicoides species on sticky traps. All treatments significantly reduced C. mississippiensis abundance compared with controls but were not different from one another (Table 4-3). Also, there was no difference in the number of Culicoides collected in Liberty Plus traps with or without the Talstar application (Table 4-4).

Significant differences in C. furens captured on sticky traps among treatments were observed $(\mathrm{F}=11.95, \mathrm{df}=3,79 \mathrm{p}<0.0001)$. The sticky surveillance trap located at the Talstar treatment area caught less $C$. furens than the control and Liberty Plus treatment areas. There were no differences between surveillance sticky traps located at the Talstar and Liberty Plus + Talstar areas. Significant differences in total C. mississippiensis captured among treatments (Control, Liberty Plus, Liberty Plus + Talstar, Talstar) from March to October 2009 were observed $(\mathrm{F}=4.45$, $\mathrm{df}=3,79 \mathrm{p}<0.0071)$. The surveillance sticky trap located at the control treatment area captured more C. mississippiensis than any surveillance sticky trap located at the other treatment areas. There were no differences in surveillance sticky traps located at the Talstar, Liberty Plus + Talstar, and Liberty Plus treatment areas.

\section{DISCUSSION}

The objective of this study was to determine if an insecticide barrier treatment alone or in conjunction with commercially available traps could provide protection against host-seeking biting midges. Providing a residual insecticide barrier around an area for protection against mosquitoes is not a novel technique (Ludvik 1950, Quarterman et al. 1955, Helson and Surgeoner 1983, Anderson et al. 1991, Perich et al. 1993, Frances 2007, Trout et al. 2007, Cilek 2008, Britch et al. 2009, Qualls et al. 2012, Bibbs et al. 2016). However, barrier treatments with a residual insecticide for protection against Culicoides has been understudied (Kettle 1949, Standfast 2003) and the author is unaware of any other study that evaluates the combination of commercial traps and barrier treatments to protect against Culicoides.

In general, all treatments provided significant reductions from biting midge pressure when compared to the control. However, the two treatments utilizing Talstar were more successful (89-98\% reduction) than the Liberty Plus trap treatment alone (68\% reduction). Previous studies have shown similar success using Talstar against mosquitoes to protect military tents (Frances 2007), park recreation areas (Cilek 2008) and even desert environments with sparse vegetation (Britch et al. 2009). In this study, the insecticide barrier treatment provided the greatest reduction in Culicoides numbers captured on the surveillance traps. The combination treatment provided the next best reduction. The Liberty Plus trap provided the least Culicoides reduction, but was still significantly better than the control treatment. Standfast (2003) reported similar success in Australia (97\% reduction) when they treated all surfaces on and surrounding the homes of their treatment sites with Bistar (bifenthrin). The authors conducted one bifenthrin application on vegetation, fence panels, and walls of the homes that they intended to protect from Culicoides. The authors monitored the population reporting a decrease in effectiveness $(60 \%$ reduction) 4-6 weeks after treatment. We were able to produce our results with treatment of the surrounding vegetation alone indicating 
that it is possible to receive near-maximum biting midge suppression with reduced insecticide exposure for up to 4 weeks.

When total Culicoides and individual species were evaluated, there were significant differences in surveillance methods used, and treatment effects. Swatting counts were not effective at assessing the host-seeking biting midge population. The short duration (3 minutes) assigned to the swatting counts was not sufficient for determining the true host seeking biting midge pressure. In addition, it is not reasonable to spend the time that seems to be required for pressure assessment. Furthermore, timing and climatic events can severely skew pressure assessments that are conducted for short periods of time. The modified sticky surveillance trap was more efficient with pressure assessment and less labor intensive. This method provided a survey for the entire time that a treatment was implemented. However, there is a substantial cost involved ( $\$ 1.50$ per kilogram) with refilling dry-ice every $24-48$ hours. The sticky surveillance trap is an efficient tool for biting midge pressure assessment, but needs to be refined to reduce costs.

The Liberty Plus trap used in the combination treatment captured more Culicoides than the Liberty Plus trap alone. It is uncertain as to why this phenomenon occurred. Although the trap capture results are not significant, it is important to discuss the pos- sibilities resulting in increased trap capture for the combination treatment. It is possible that the insecticide treatment dramatically reduced the amount of resting harborage available due to behavioral avoidance; therefore the Culicoides will be more likely to fly towards a trap that produces an attractant plume rather than rest on a surface treated with insecticide, creating a push-pull protection system. Another possibility is the addition of the Liberty Plus trap in combination with the insecticide treatment is attracting or pulling in the biting midges from outside the protected area actually attracting biting midges that may not have normally traveled to the home.

The results from this study suggest that the Liberty Plus trap, Talstar and Talstar/ Liberty Plus (combination) treatment will reduce the Culicoides population pressure around homes. However, to maximize suppression and response time for protection from Culicoides, insecticide treatment alone is the most efficient and economically effective population management technique. Commercial traps may have potential for long term (3-5 years) control programs by providing an alternative control solution to manage insecticide resistance and potentially decreasing a pestiferous population over time. Further evaluations utilizing insecticide applications and commercial traps as a combination treatment are needed.

Table 4-1. Total number of Culicoides species caught in Cedar Key, FL. from March to October 2009 using four modified sticky surveillance traps and swatting counts.

\begin{tabular}{lccc}
\hline \hline Species & Sticky surveillance traps & Swatting paper & \% of total Culicoides captured \\
C. furens & 56,779 & 1,709 & 88.85 \\
C. mississippiensis & 6,328 & 251 & 9.90 \\
C. barbosai & 532 & 0 & 0.84 \\
C. melleus & 262 & 0 & 0.41 \\
Grand Total & 63,901 & 1,960 & 100 \\
\hline
\end{tabular}

Table 4-2. Total number of Culicoides species caught in two MM-Liberty Plus traps in Cedar Key, FL from March through October 2009. $\mathrm{n}=60$

\begin{tabular}{lcc}
\hline \hline Species & No. of Culicoides spp. captured & \% of total Culicoides Captured \\
\hline C. furens & 77,910 & 92.63 \\
C. mississippiensis & 4,898 & 5.82 \\
C. barbosai & 843 & 1.02 \\
C. melleus & 449 & 0.53 \\
Grand Total & 84,100 & 100 \\
\hline
\end{tabular}


Table 4-3. Mean number of Culicoides species captured on modified sticky surveillance traps placed at each treatment site in Cedar Key, FL from March through October 2009. n = 80; $<<0.05$

\begin{tabular}{lccc}
\hline \hline Treatments & Total Culicoides & Culicoides furens & Culicoides mississippiensis \\
\hline Control & $2024 \pm 440 \mathrm{a}$ & $1905 \pm 411 \mathrm{a}$ & $890 \pm 325 \mathrm{a}$ \\
Liberty Plus & $864 \pm 322 \mathrm{~b}$ & $234 \pm 79 \mathrm{~b}$ & $205 \pm 79 \mathrm{~b}$ \\
Liberty Plus + Tastar & $45 \pm 13 \mathrm{bc}$ & $38 \pm 11 \mathrm{bc}$ & $119 \pm 46 \mathrm{~b}$ \\
Talstar & $25 \pm 11 \mathrm{c}$ & $29 \pm 20 \mathrm{c}$ & $6 \pm 3 \mathrm{~b}$ \\
\hline
\end{tabular}

Within a column, means followed by the same letter designate no significant difference 223 in collections among treatments (alpha $=0.05$ ).

$\mathrm{n}=$ the number of trap replicates for the corresponding time period.

Within a column, degree of freedom for species captured error for each treatment is 79 ; treatment $\mathrm{df}=3$

Data were $\log (\mathrm{n}+1)$ transformed prior to analysis using an ANOVA with trapping method (sticky surveillance trap)and treatment in the model. Significance among treatments analyzed with Ryan-Einot-Gabriel-Welsch multiple range test.

Table 4-4. Mean trap capture comparison between the MM-Liberty Plus and MM-Liberty Plus+ Talstar treatments in Cedar Key, FL from March through October 2009. $n=60 ; \mathrm{p}<0.05$

\begin{tabular}{lcc}
\hline \hline Species & MM-Liberty Plus & MM-Liberty Plus+ Talstar \\
\hline Culicoides spp. & $1,972 \pm 520$ & $2,232 \pm 462$ \\
C. furens & $1,948 \pm 516$ & $2,184 \pm 460$ \\
C. mississippiensis & $24 \pm 14$ & $48 \pm 23$ \\
\hline
\end{tabular}

Within a row, there were no significant differences between MM-Liberty Plus trap collection means (alpha=0.05).

$n=$ the number of trap replicates for the corresponding time period.

Within a column, degree of freedom for species captured error is 59; trap $\mathrm{df}=1$.

Data were $\log (n+1)$ transformed prior to analysis using an ANOVA with trapping period (method) and trap treatment in the model. Significance between traps analyzed with Ryan-Einot-Gabriel-Welsch multiple range test.

\section{REFERENCES CITED}

Allan SA, Kline DL, Walker T. 2009. Environmental factors affecting efficacy of bifenthrin-treated vegetation for mosquito control. J Am Mosq Control Assoc. 25:338-346.

Anderson AL, Apperson CS, Knake R. 1991. Effectiveness of mist-blower applications of malathion and permethrin to foliage as barrier sprays for salt-marsh mosquitoes. JAm Mosq Control Assoc. 7:116-117.

Bazin M, Williams CR. 2018. Mosquito traps for urban surveillance collection efficacy and potential for use by citizen scientists. J Vector Ecol. 43:98-103

Bibbs C, Anderson, C, Smith ML, Xue RD. 2016. Bifenthrin barrier spray against Aedes albopictus around an urban cemetery, St. Augustine, Florida. Tech Bull Fla Mosq Control Assoc. 10:76-80.

Blanton FS, Wirth WW. 1979. The sand flies (Culicoides) of Florida (Diptera: Ceratopogonidae). Vol. 10 in Arthropods of Florida, neighboring land areas. Tallahassee, FL: Florida Department of Agriculture Consumer Service. 204 p.

Breidenbaugh MS, Szalay FA. 2010. Effects of aerial applications of Naled on nontarget insects at Parris Island, South Carolina. Environ Entomol. 39:591-599.

Britch SC, Linthicum KJ, Wynn WW, Walker TW, Farooq M, Smith VL, Robinson CA, Lothrop BB, Snelling M, Gutierrez A, Lothrop HD. 2009. Evaluation of barrier treatments on native vegetation in a southern California desert habitat. J Am Mosq Control Assoc. 25:184-193.

Bruce WG, Blakeslee EB. 1948. Control of salt-marsh sand flies and mosquitoes with DDT insecticides. Mosq. News 8:26-27.
Cilek JE. 2008. Application of insecticides to vegetation as barriers against host-seeking mosquitoes. $\mathrm{J} \mathrm{Am}$ Mosq Control Assoc. 24:172-176.

Floore TG. 1985. Laboratory wind tunnel tests of nine insecticides against adult Culicoides species. Fla Entomol. 68:678-682.

Frances SP. 2007. Evaluation of bifenthrin and permethrin as barrier treatments for military tents against mosquitoes in Queensland, Australia. $J \mathrm{Am}$ Mosq Control Assoc. 23:208-212.

Fulcher A, Farooq M, Smith ML, Li C, Scott JM, Thompson E, Kaufman PE, Xue RD. 2015. Evaluation of a new spraying machine for barrier treatment and penetration of bifenthrin on vegetation against mosquitoes. JAm Mosq Control Assoc. 31:85-92.

Giglioli ME, Gerberg EJ, Todd RG. 1980. Large scale field tests and environmental assessments of Sumithion ${ }^{\circledR}$ (fenitrothion) against adult biting midges in Grand Cayman, West Indies. Mosq. News 40:1-5.

Haile DG, Kline DL, Reinert JF, Biery TL. 1984. Effects of aerial applications of Naled on Culicoides biting midges, mosquitoes and tabanids on Parris, Island SC. Mosq. News. 44:178-183.

Helson BV, Surgeoner GA. 1983. Permethrin as a residual lawn spray for adult mosquito control. Mosq News. 43:164-169.

Kettle DS. 1949. An attempt to control Culicoides impunctatus Goetghebuer in Scotland by barrier spraying. Ann Trop Med Parasit. 43:284-296.

Kline DL, Haile DG, Baldwin KF. 1981. Wind tunnel tests with seven insecticides against adult Culicoides mississippiensis Hoffman. Mosq News. 41:745-747.

Kline DL. 2006. Traps and trapping techniques for adult mosquito control. J Am Mosq Control Assoc. 22:490-496. 
Li Y, Su X, Zhou G, Zhang H, Puthiyakunnon S, Shuai S, Cai S, Zhou X, Yan G, Chen XG. 2016. Comparative evaluation of the efficiency of the BG-Sentinel trap, CDC light trap and Mosquito-oviposition trap for the surveillance of vector mosquitoes. Parasites and Vectors 9, 446. https://doi.org/10.1186/s13071016-1724-x

Linley JR, Davies JB. 1971. Sandflies and tourism in Florida and the Bahamas and Caribbean Area.JEcon Entomol. 64:264-278.

Linley JR, Jordan S. 1992. Effects of ultra-low volume and thermal fog malathion, Scourge ${ }^{\circledR}$ and naled applied against caged adult Culicoides furens and Culex quinquefasciatus in open and vegetated terrain. $\mathrm{J} \mathrm{Am}$ Mosq Control Assoc. 8:69-76.

Lloyd AM, Kline DL, Hogsette JA, Kaufman PE, Allan SA. 2008. Evaluation of two commercial traps for the collection of Culicoides (Diptera: Ceratopogonidae). J Am Mosq Control Assoc. 24:253-262.

Ludvik GF. 1950. Barrier strip and pre-flood treatments with DDT to control Anopheles quadrimaculatus. J Econ Entornol. 43:516-519.

Madden AH, Lindquist AW, Langcoy OM, Knipling EF. 1946. Control of adult sand flies by airplane spraying with DDT. Fla Entomol. 29:5-10.

Perich MJ, Tidwell MA, Dobson SE, Sardelis MR, Zaglul A, Williams DC. 1993. Barrier spraying to control the malaria vector Anopheles albimanus: laboratory and field evaluation in the Dominican Republic. Med Vet Entomol. 7:363-368.

Qualls WA, Smith ML, Muller GC, Zhao TY, Xue RD. 2012. Field evaluation of a large-scale barrier application of bifenthrin on a golf course to control floodwater mosquitoes. JAm Mosq Control Assoc. 28:219-224.
Quarterman KD, Jensen JA, Mathis W, Smith WW. 1955. Barrier-strip spraying for the control of rice field mosquitoes. JEcon Entomol. 48:107-108.

Richards SL, Volkan JK, Balanay JG, Vandock K. 2017. Evaluation of bifenthrin and deltamethrin barrier sprays for mosquito control in eastern North Carolina. J Med Entomol. 54:1659-1665.

SAS Institute. 2003. User's guide, statistics. Version 9.1. Cary, NC: SAS Institute.

Standfast H, Fanning I, Maloney L, Purdie D, Brown M. 2003. Field evaluation of Bistar ${ }^{\circledR}$ environmental health insecticide as an effective insecticide harborage treatment for biting midges (Culicoides) and mosquitoes infesting peridomestic situations in an urban environment. Bull Mosq Control Assoc. 15:1-11.

Trapido H. 1947. DDT residual spray control of sand flies in Panama. JEcon Entomol. 40:472-475.

Trout RT, Brown GC, Potter MF, Hubbard JL. 2007. Efficacy of two pyrethroid insecticides applied as barrier treatments for managing mosquito (Diptera:Culicidae) populations in suburban residential properties. J Med Entomol. 44:470-474.

Wilke ABB, Carvajal A, Medina J, Anderson M, Nieves VJ, Ramirez M, Vasquez C, Petrie W, Cardenas G, Beier JC. 2019. Assessment of the effectiveness of BG-Sentinel traps baited with CO2 and BG-Lure for the surveillance of vector mosquitoes in MiamiDade County, Florida. PLoS One. 2019; 14(2): e0212688. doi: 10.1371/journal.pone. 0212688 PMCID: PMC6386269

World Health Organization. 2013. Handbook for integrated vector management. Outlooks Pest Manag. 24: 142-143. 